\title{
Frictional characteristics of molecular length ultra-thin boundary adsorbed films
}

\author{
I.S.Y. Ku ${ }^{1}$, W.W.F. Chong ${ }^{1,2^{\star}}$, T. Reddyhoff ${ }^{3}$, H. Rahnejat ${ }^{4}$ \\ 1 National Centre for Advanced Tribology (nCATS), Faculty of Engineering and the Environment, University of \\ Southampton, Southampton, UK \\ ${ }_{2}$ Faculty of Engineering and the Environment, University of Southampton Malaysia Campus, Nusajaya, Johor, \\ Malaysia \\ ${ }^{3}$ Tribology Group, Department of Mechanical Engineering, Imperial College London, London, UK \\ ${ }^{4}$ Wolfson School of Mechanical \& Manufacturing Engineering, Loughborough University, Loughborough, UK \\ ${ }^{*}$ Corresponding Author \\ Email: w.chong@soton.ac.uk
}

\begin{abstract}
The paper presents measurements of friction of any ultra-thin film entrained into the contact of a pair of very smooth specimen subjected to entrainment in a converging micro-wedge of a special-purpose micro-tribometer. An ultra-thin film is expected to form at the boundary solids through adsorption of boundary active molecules. Fluids with linear and branched molecules are used in the investigation. It is found that the frictional characteristics of these films can be adequately described through use of Eyring thermal activation energy and a potential energy barrier to sustain conjunctional sliding motion. The combined experimental measurement and the simple activation energy approach shows that the thin molecular adsorbed films act like hydroLangmuir-Blodgett layers, the formation and frictional characteristics of which are affected by the competing mechanisms of adsorption, forced molecular re-ordering and discrete-fashion drainage through the contact by the solvation effect. This process is a complex function of the contact sliding velocity as well as a defined Eyring activation density (packing density of the molecules within the conjunction). It is shown that the contribution of solvation to friction is in the form of energy expended to eject layers of lubricant out of the contact, which unlike the case of micro-scale hydrodynamic films, is not a function of the sliding velocity.
\end{abstract}

Keywords: ultra-thin boundary films, thermal activation energy, solvation, friction

\subsection{Introduction}

Ultra-thin adsorbed boundary films play a dominant role in the smooth running of many conjunctions; in applications ranging from boundary lubricated engineering components to Microelectromechanical Systems (MEMS) to biological interfaces. These ultra-thin adsorbed films form as the result of tribo-chemical and physical interactions between the lubricant molecules and the surface material. They exhibit low shear strength characteristics. Unlike hydrodynamic films formed through viscous action of bulk lubricant, the knowledge concerning the underlying mechanisms of thin adsorbed films is still emerging. These thin films form as the result of intermolecular interactions between fluid molecular species, as well as between these and the atoms of the bounding conjunctional solid surfaces. Therefore, it is necessary to gain a better understanding of the plethora of kinetic laws that belie such interactions.

In nano-scale, the packing order of the fluid molecules near solid barriers varies because of the free surface energy and intermolecular interactions of competing kinetic nature. The kinetic interactions in diminishing gaps typically comprise long range van der Waals interactions between fluid molecular species and the solid barriers [1]. For polar fluids and charged surfaces, electrostatic interactions usually prevail in small separations [2]. It is also often recognized that the fluid molecular density is higher near solid barriers when compared with that in the bulk [3]. As a result, mutual convergence of fairly smooth bounding surfaces induces an oscillatory attractive-repulsive force, termed solvation [3,4]. The oscillatory solvation forces arise from the variation in the packing density of molecules, confined between solid barriers [3]. During the approach of solid barriers, the molecules are ordered into distinct layers in a step-wise fashion. A periodic oscillatory force profile ensues with discrete layers of molecules ejected out of the conjunction [5]. The downside of solvation 
is that the oscillation as a result of molecular restructuring can induce vibrations within the contact especially in diminishing gaps of molecular length scale, which might affect the performance of micro-mechanisms.

Tabor and Winterton developed one of the first apparatus, known as the surface force apparatus (SFA), to measure kinetic interactions in nano-scale [6]. Using the SFA, Horn and Israelachvili [7] and Chan and Horn [8] measured the oscillatory force between very smooth mica surfaces in liquids such as Octamethyltetracyclosiloxane (OMCTS), Hexadecane and Tetradecane. In a more recent work, Lim and O'Shea [9] used an atomic force microscope (AFM) to measure solvation of fluid such as Hexadenane and Squalane. A point to note is that the aforementioned force profiles are all measured normal to the bounding surfaces.

Based on the concept of molecular oscillation for confined fluids, various mathematical models have been developed, integrating an amalgam of kinetic laws ranging from hydrodynamics to surface energy effects and also intermolecular interactions. They include the works reported by Matsuoka and Kato [10], Al-Samieh and Rahnejat $[11,12,13]$ and Chong et al [14] for the study of lightly loaded conjunctions with diminutively thin films, entrained through by hydrodynamic viscous action. To describe solvation, Chong et al $[15,16]$ also adopted the use of Ornstein-Zernike (OZ) equation as expounded by Mitchel et al [17], Henderson and Lozada-Cassou [18] and Attard and Parker [19].

The SFA was further developed by Israelachvilli [20] to measure friction along diminishing gaps. Through this, Israelachvilli established that friction at nano-scale could be present at the sliding crystalline interface in the absence of material wear. Later, Israelachvilli et al [21] found that friction at such a scale might be correlated with adhesion hysteresis rather than the mechanism of adhesion itself. This finding ushered an era of investigations of mechanisms governing friction at nano-scale interfaces, especially those separated by a molecularly thin adsorbed film of any lubricant molecular species [22,23,24]. More recent attempts to understand the formation of boundary fluid films include measuring the film thickness within confined nano-gap using optical interference intensity method $[25,26]$.

Chong et al [14] predicted a step-wise friction for OMCTS with increasing applied loads. Through numerical analysis, they observed that with an increasing sliding velocity, the step-wise effect of molecular discretization diminished. The oscillatory solvation is rarely noted at the micro-scale, especially at slow sliding speeds. Therefore, based on the modified Eyring thermal activation approach, the current study attempts to investigate the solvation phenomenon under slow sliding speeds using a micro-tribometer developed by Ku et al [27].

\subsection{Experimental Approach}

The experimental work was carried out with a micro-tribometer developed by Ku et al [27]. The set up comprises two $2 \mathrm{~mm}$ diameter silicon specimens, one stationary, with a surface pattern similar to that of a step bearing (see figure 1), whilst the other is a polished rotating surface (with an $R_{a}$ roughness of $0.65-0.75 \mathrm{~nm}$ ). The step-bearing pattern was chosen as the experiment also aimed to explore friction in the hydrodynamic regime, which requires a converging surface geometry. A thin layer of native oxide exists on the mating surfaces. The authors did not have the means to accurately measure this thickness, but the oxide is believed to be between 2-3 nm according to Gavrilenko et al [28]. The rotating specimen is mounted at the end of a motor shaft (on a motorized stage) via a self-aligning mechanism. This mechanism consists of a ball and two-pin design (see figure 2), where the upper specimen is held onto the end of the shaft by the nickel-plated backside of the specimen. This enables the upper specimen to pivot about the ball to ensure that the specimens are parallel to each other when brought into contact. 

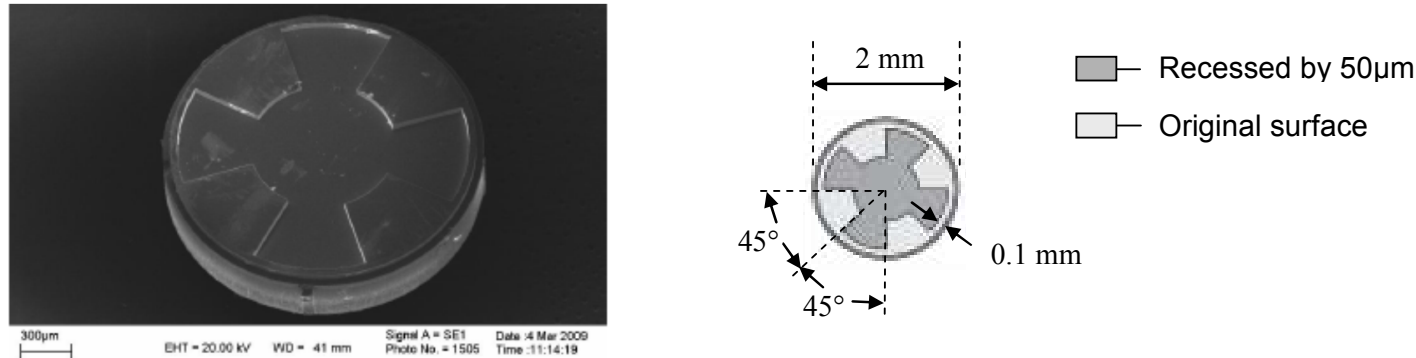

Figure 1. SEM image and a sketch of the lower stationary specimen (reproduced from [29])

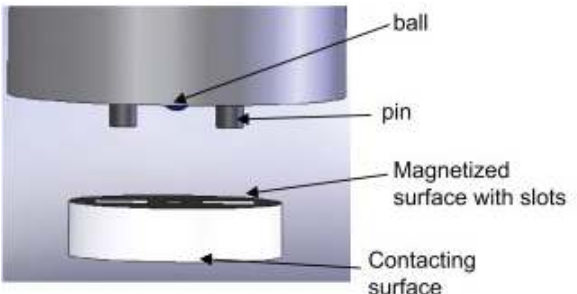

(a)

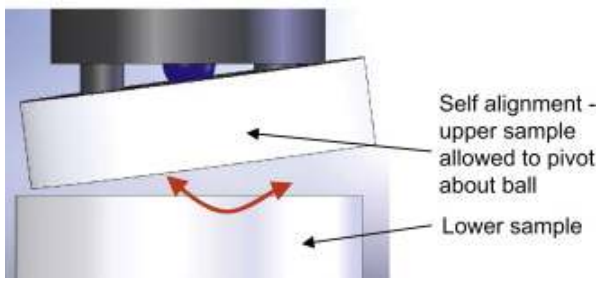

(b)

Figure 2. Self-alignment mechanism, reproduced from [27]

The stationary specimen and its holder, along with an optional lubricant bath are located on a platform. This consists of two parts, providing known stiffness contributions in the vertical and rotational directions (see figure 3). Their displacements during an experiment determine the normal and frictional forces respectively.

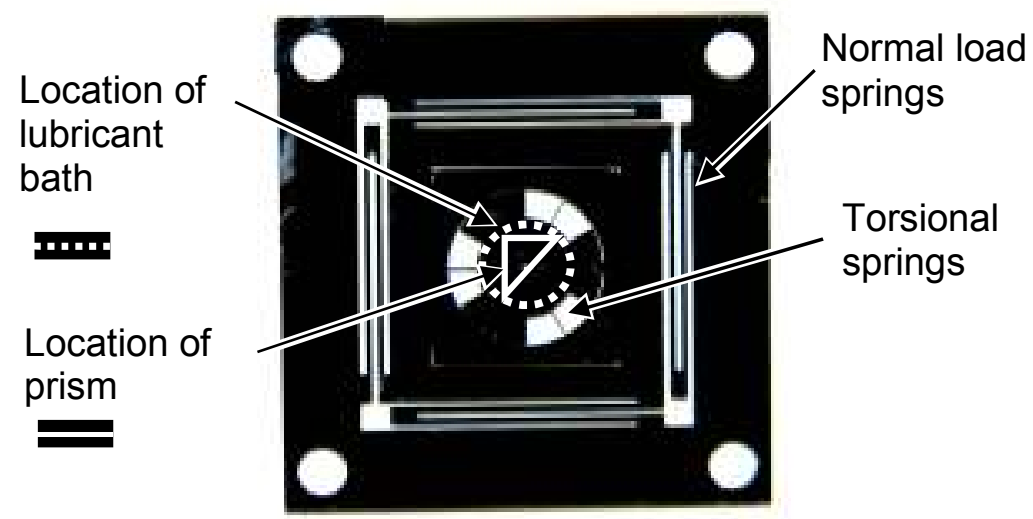

Figure 3. Close up of the test platform, showing the inner platform with the torsional springs, the outer platform with the normal load springs, the locations for the lubricant bath and the prism [30]

The upper rotating specimen, attached to a high precision motor-controlled linear stage, is lowered down onto the stationary specimen, residing on its platform. A laser displacement measurement device is mounted under the platform in order to measure any vertical displacements. The normal load is then applied by lowering the stationary specimen further until the desired displacement (load) of the platform is reached. The rotational displacement is measured by a custom optical lever system. The system comprises a prism located beneath the platform and a series of mirrors, which lengthen the laser beam, making it possible to measure the small rotational displacements by a detector. A piezo-drive is mounted onto a mirror and connected to a feedback system, which rotates the mirror and drives the laser beam to the centre of the detector (see figure 4). A compensating voltage is required, which provides a measure of the rotational displacement of the lower specimen. From this, the frictional torque can be calculated. Overall, the instrument can operate at speeds up to $20,000 \mathrm{rpm}$, at a normal load range of 0 to $0.2 \mathrm{~N}$. The range of measurable friction coefficient depends on the test platform, as the stiffnesses can be varied by altering 
the flexure dimensions. So far, it can successfully measure as low as $0.005 \mathrm{~N}$ up to as high as 3 to $4 \mathrm{~N}$.

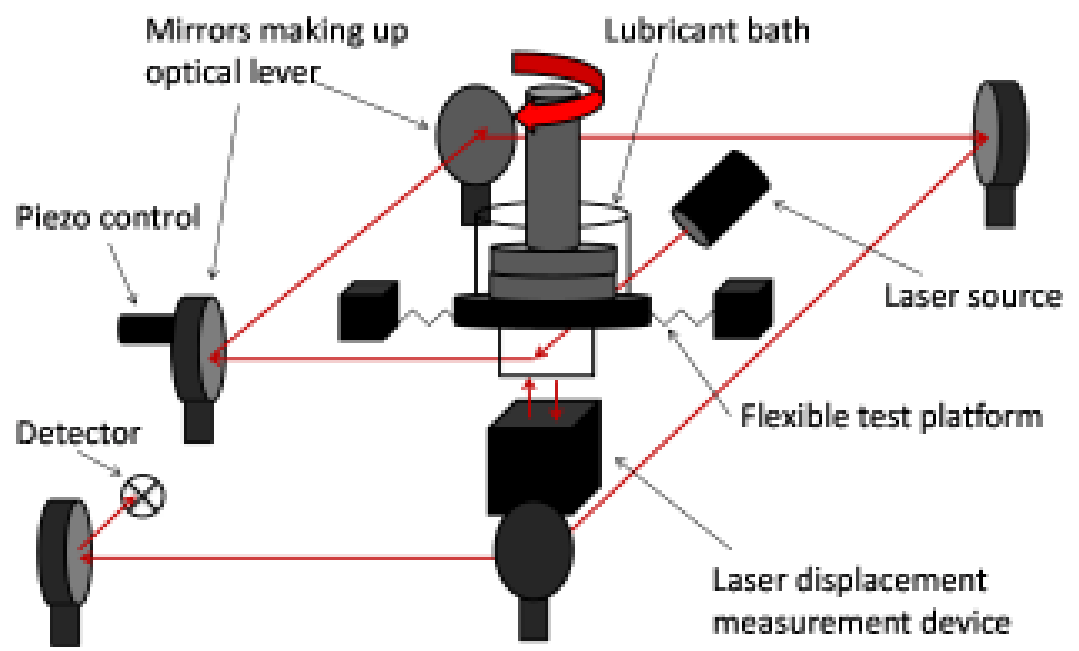

Figure 4 Schematic setup for the micro-tribometer

The experiments are carried out in a temperature controlled room held at $25^{\circ} \mathrm{C}$. There is no formal temperature control at the rig itself, but the size of the lubricant bath is large compared to the specimens, and hence is sufficient to dissipate the small amount of heat generated. The experimental process commences by activating the interfacing software, which is written to automate the test process and acquire the test data. For the purpose of the current investigation, the test program involves running a series of "Stribeck curve" tests, where a constant load is applied. Both specimens are fully lubricated by a neat liquid, as indicated in the results, with the friction readings taken at various rotational speeds.

\subsection{Mathematical Approach}

Using the Eyring thermal activation theory, in a sliding process, it is assumed that the average time to overcome a single molecular barrier through shear conforms to a Boltzmann distribution. When a regular series of such barriers are encountered, the Eyring shear stress can be defined as [31,32]:

$$
\tau=\frac{k_{B} T}{\phi} \ln \left(\frac{v}{v_{0}}\right)+\frac{1}{\phi}(Q+P \Omega)
$$

The potential energy barrier, $E$, required to overcome the sliding process (see figure 5), is given as:

$$
E=Q+P \Omega-\tau \phi
$$

where $\Omega$ is the pressure activation volume, $\phi$ the shear stress activation volume, $Q$ the activation energy to initiate the sliding process, $P$ the applied normal pressure and $\tau$ the shear stress. Briscoe and Evans [31] used the SFA to obtain the potential energy barrier, $E$. Through the experiment, assuming a constant sliding velocity, $v$ and temperature, $T$, Briscoe and Evans [31] simplified equation (1) in order to describe the shear stress as a function of the varying applied normal pressure as: 


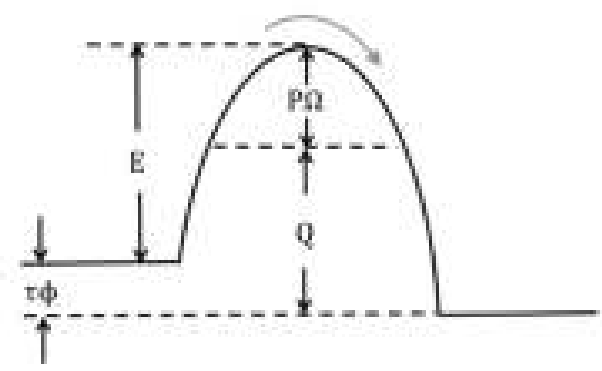

Figure 5. Potential barrier based on Eyring thermal activation energy

$$
\tau=\tau_{0}+\xi P
$$

where: $\tau_{0}=\frac{1}{\phi}\left[k_{B} T \ln \left(\frac{v}{v_{0}}\right)+Q\right]$ and $\xi=\frac{\Omega}{\phi}$

Applying a similar approach for an assumed constant normal pressure, $P$ and temperature, $T$, the expression relating the shear stress to the sliding velocity of the contact can be obtained as [31]:

$$
\tau=\tau_{1}+\theta \ln v
$$

where: $\tau_{1}=\frac{1}{\phi}\left[Q+P \Omega-k_{B} T \ln v\right]$ and $\theta=\frac{k_{B} T}{\phi}$.

\subsection{Results and Discussions}

The frictional characteristics for hexadecane (with linear chain molecules) and squalane (with branched molecules) are measured using the micro-tribometer described above, where the contacts are fully submerged, with an applied load of $0.05 \mathrm{~N}$. Friction curves obtained are shown in figure 6(a) and figure 7(a). As the sliding velocity is increased there is an initial fall in the stress. This may be because of a gradual formation of an adsorbed film to the surfaces. It is also interesting to note a slight negative shear stress for squalane (see figure 6(a)), which might be because of attractive contact forces that result from the inability of the initial layer of squalane molecules to form an adsorbed layer [3]. Thereafter, there is an increasing shear stress proportional to the natural logarithm of the sliding velocity, which is unlike hydrodynamic viscous shear (occurring at speed above $12 \ln (\mu \mathrm{m} / \mathrm{s}$ ) for both hexadecane and squalane), where typically: $\tau \propto v$. The increasing shear stress is an indication of a growing adsorbed film (a greater packing density of lubricant molecules). Subsequently, some oscillatory behaviour can be observed with an increasing shear. This may be as the result of molecular re-ordering and thin film discretization. However, this observation is solely based on friction and may not suffice to conclude the presence of solvation. For speeds above approximately $10 \ln (\mu \mathrm{m} / \mathrm{s})$, the shear stress drops as the contact enters the mixed lubrication regime, in which part of the load is carried by hydrodynamic support. Then, for speeds above approximately $12 \ln (\mu \mathrm{m} / \mathrm{s})$, the contact is fully flooded and friction rises with speed due to an increased shear rate. From the experimental data, the Eyring activation energies are computed for hexadecane and squalane at various speeds with constant loading. The components contributing to the height barrier, $E$ comprise the process activation energy, $Q$, pressure activation energy, $P \Omega$ and shear activation energy, $\tau \phi$. Here, to calculate $\theta$ from the measured friction data, equation (4) has to be modified. The term $\theta$ is redefined as the instantaneous rate of change of friction (i.e. friction hysteresis) at different sliding velocities:

$$
\theta=\frac{d \tau}{d(\ln v)}
$$

where, $\tau$ is in a function of $\ln v$, based on curve fitted equations for the friction data measured for hexadecane and squalane. Taking $\xi=0.23$ for hexadecane and $\xi=0.33$ for squalane (obtained from the friction force-normal load measurements), the height barrier for the 
Eyring's "Cage" model, $E$ can be calculated through simultaneous solution of equations (3) and (4). Referring to figure $6(\mathrm{~b})$ and figure $7(\mathrm{~b})$, it can be seen that the energy barrier, $E$ remains almost linear with a negative slope for both hexadecane and squalane. These show that a progressively diminishing barrier is experienced with the continuance of sliding motion. The reducing barriers can be as the result of the fluid molecules adsorbing to the opposing surfaces during sliding, forming a thin boundary film, which would decrease the boundary shear strength.

The process activation energy, $Q$ shows a distinctive oscillatory behaviour for both the fluids. However, the solvation phenomenon is closely related to the density variation within the confined area, which still cannot be explicitly related to the process activation energy. Therefore, by inverting the activation volumes $\Omega$ and $\phi$, the activation densities can be obtained in the units of $\mathrm{mol} / \mathrm{cm}^{3}$ (figure 6(c) and figure 7(c)). From the pressure activation density, $1 / \Omega$, it is evident that the oscillatory characteristics of solvation prevails for hexadecane, which is evidenced by $\mathrm{He}$ et al [32], showing interfacial liquid structuring during shearing of simple fluids like hexadecane. This is also in accord with Chan and Horn's [8] observation that fluids with simple molecules (e.g. spherical and linear chain molecules) tend to solvate near fairly smooth solid barriers. The same appears to be true for squalane as shown in figure 7(c). This observation further validates the measurements of Granick et al [33] (using SFA) and lately by Lim and O'Shea [34] (using AFM), showing oscillatory forces exist for branched molecules such as squalane and 3-methylundecane $\left(\mathrm{C}_{12} \mathrm{H}_{26}\right)$.

The pressure activation density, $1 / \Omega$ only represents the normal reaction of the fluid molecules towards the bounding surfaces. However, in sliding motion the focus is directed to the shearing of molecules, which is the main contribution to contact friction. Therefore, it is also critical to observe the change in the shear stress activation density, $1 / \phi$. This activation density can be interpreted as the dislocation volume in the tangential direction for the unit shearing process, which is attributed to a molecule or a dislocation line [32]. The oscillatory characteristics observed for both hexadecane and squalane clearly indicate the existence of molecular restructuring during shearing of the fluid molecules especially at slow sliding speeds. It can also be observed that hexadecane exhibits a larger oscillatory characteristic when compared with squalane. Squalane with a branched and irregularly shaped molecules, would tend not to re-order easily into discrete layers within a confined space, thus exhibit a reduced oscillatory behaviour [3]. A negative activation density, $1 / \phi$ exists at these speeds because of the energy expended to eject rows of molecules from the contact (fluid layer discretisation, which is a characteristic of the solvation effect). It can be seen that the oscillatory response ceases when a critical sliding velocity is reached, corresponding to a parabolic increase in activation densities. This phenomenon heralds a hydrodynamic effect at higher sliding velocities with the onset of uniform motion (laminar flow). Therefore, at a critical sliding velocity, with all other conditions remaining unchanged there is a transition from boundary lubrication of thin adsorbed films to hydrodynamic lubrication, where bulk rheological properties of the fluid become dominant [30].

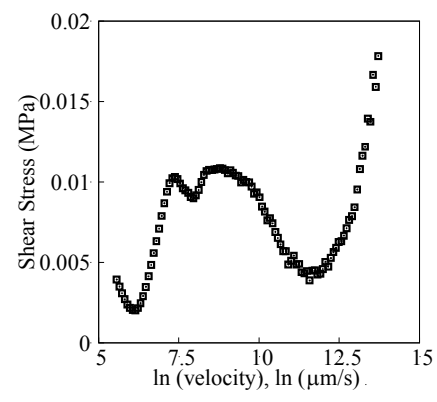

(a) Shear Stress

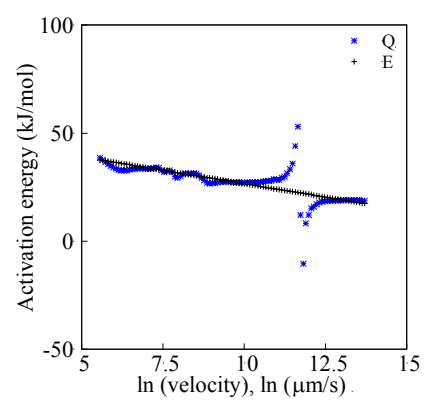

(b) Eyring activation energies

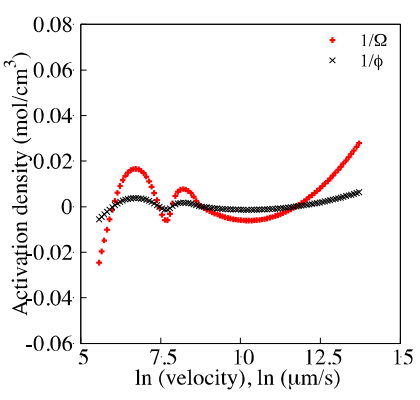

(c) Eyring activation densities

Figure 6 Frictional characteristics for Hexadecane 


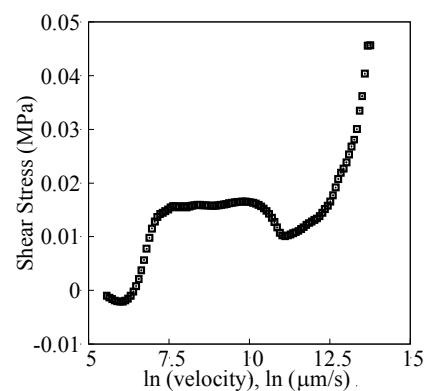

(a) Shear Stress

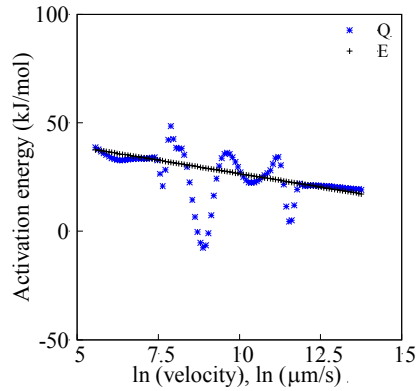

(b) Eyring activation energies

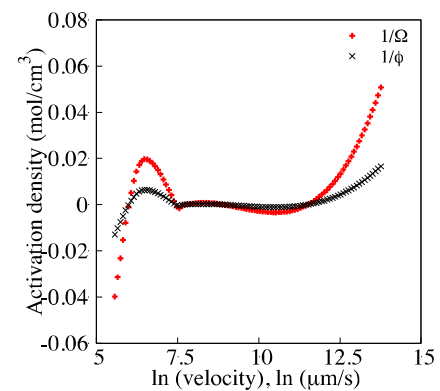

(c) Eyring activation densities

Figure 7 Frictional characteristics for Squalane

\subsection{Conclusions}

A series of experiments are reported with a micro-tribometer to study the boundary frictional characteristics of linear chain and branched molecules. The aim of the investigation was to ascertain the influence of solvation on the formation of an adsorbed ultra-thin film on fairly smooth surfaces (such as at the summit of ubiquitous asperities) in diminishing gaps of molecular length scale. Using Eyring's thermal activation theory with a potential energy barrier, the presence of solvation is noted, which conforms to the shear characteristics of a Langmuir-Blodgett layer as described by Briscoe and Evans [31]. Unlike the hydrodynamic viscous shear of micro-scale films, solvation affects friction characteristics as an energy sink through thin film discretization and ejection of layers of lubricant from the conjunction. This effect depends on the packing density of the molecules within the conjunction, which is a complex function of the sliding velocity, and seems to be only effective over a range of speeds (molecular layers), before a bulk lubricant film of hydrodynamic characteristics is formed. This finding is in line with the experimental works in $[7,8,9,34]$ and complex numerical analysis, such as that for a progressively diminishing gap by Teodorescu et al [35]. The main contribution of this paper is in demonstrating that such characteristics can be predicted using a relatively simple analytical approach, based on the assumption of a Langmuir-Blodgett layer subjected to Eyring thermal activation energies.

\section{Acknowledgements}

The authors acknowledge sponsorship provided by the EPSRC through Grant Numbers EP/D04099X and EP/L001624/1 (covering experimental work) along with the EPSRC ENCYCLOPAEDIC program grant (covering theoretical work).

\section{References}

[1] E. M. Lifshitz, "The theory of molecular attractive forces between solids," Soviet Physics JETP, vol. 2, pp. 94-110, 1956.

[2] R. Gohar and H. Rahnejat, Fundamentals of tribology, Imperial College Press, London, 2008.

[3] J. Israelachvili., Intermolecular and surface forces, 2nd ed. New York: Academic Press, 1992.

[4] P. Attard and J. L. Parker, "Oscillatory solvation forces: A comparison of theory and experiment," J. Phys. Chem., vol. 96, no. 12, pp. 5086-5093, 1992.

[5] R. Lim and S. J. O'Shea., "Solvation forces in branched molecular liquids," Phys. Rev. Lett., vol. 88, no. 24, p. 246101, 2002.

[6] D. Tabor and R. H. S. Winterton, "Surface forces: Direct measurement of normal and retarded van der waals forces ," Nature, vol. 219, pp. 1120-1121, 1968.

[7] R. G. Horn and J. N. Israelachvili, "Direct measurement of structural forces between two surfaces in a nonpolar liquid," J. Chem. Phys., vol. 75, no. 3, pp. 1400-1412, 1981.

[8] D. Y. C. Chan and R. G. Horn, "The drainage of thin liquid films between solid surfaces," 
J. Chem. Phys., vol. 83, no. 10, pp. 5311-5324, 1985.

[9] R. Y. H. Lim and S. J. O'Shea, "Discrete solvation layering in confined binary liquids," Langmuir, vol. 20, no. 12, pp. 4916-4919, 2004.

[10] H. Matsuoka and T. Kato, "An ultrathin liquid film lubrication theory - calculation method of solvation pressure and its application to the EHL problem," J. Trib., vol. 119, pp. 217226, 1997.

[11] M. Al-Samieh and H. Rahnejat, "Ultra-thin lubricating films under transient conditions," J. Phys D: App. Phys., vol. 34, pp. 2610-2621, 2001.

[12] M. F. Abd. Al-Samieh and H. Rahnejat, "Nano-lubricant film formation due to combined elastohydrodynamics and surface force action under isothermal conditions," Proc. IMechE, Part C : J. Mech. Eng. Sci., vol. 215, pp. 1019-1029, 2001.

[13] M. Al-Samieh and H. Rahnejat, "Physics of lubricated impact of a sphere in a plate in a narrow continuum to gaps of molecular dimensions," J. Phys D: App. Phys, vol. 35, pp. 2311-2326, 2002.

[14] W. W. F. Chong, M. Teodorescu, and H. Rahnejat, "Effect of lubricant molecular rheology on formation and shear of ultra-thin surface films," J. Phys D : App. Phys, vol. 44, no. 16, p. 165302, 2011.

[15] W. W. F. Chong, M. Teodorescu, and H. Rahnejat, "Formation of ultra-thin bi-molecular boundary adsorbed films," J. Phys., D: Appl. Phys, vol. 45, no. 11, p. 115303, 2012.

[16] W. W. F. Chong, M. Teodorescu, and H. Rahnejat, "Physio-chemical hydrodynamic mechanism underlying the formation of thin adsorbed boundary films," Faraday Discuss, vol. 156, pp. 123-136, 2012.

[17] D. J. Mitchell, B. W. Ninham, and B. A. B. A. Pailthorpe, "Hard sphere structural effects in colloid systems," Chem. Phys Lett, vol. 51, no. 2, pp. 257-260, 1977.

[18] D. Henderson and M. Lozada-Cassou, "A simple theory for the force between spheres immersed in a fluid," J. Coll. Inter. Sci, vol. 114, no. 1, pp. 180-183, 1986.

[19] P. Attard and J. L. Parker, "Oscillatory solvation forces: A comparison of theory and experiment," J. Phy. Chem, vol. 96, no. 12, pp. 5086-5093, 1992.

[20] J. N. Israelachvili, "Thin-film studies using multiple-beam interferometry," J. Colloid Interface Sci., vol. 44, p. 259272, 1973.

[21] H. Yoshizawa, Y. L. Chen, and J. N. Israelachvili, "Fundamental mechanisms of interfacial friction. 1. Relation between adhesion and friction," J. Phys. Chem., vol. 97, p. 41284140, 1993.

[22] J. N. Israelachvili and D. Tabor, "Shear properties of molecular films," Nat. Phys. Sci., vol. 241, p. 148149, 1993.

[23] H. Yoshizawa, Y. L. Chen, and J. N. Israelachvili, "Recent advances in molecular understanding of adhesion, friction and lubrication ," Wear, vol. 168, pp. 161-166, 1993.

[24] H. Yoshizawa and J. N. Israelachvili, "Fundamental mechanisms of interfacial friction.2.stickslip friction of spherical and chain molecules ," J. Phys. Chem. , vol. 97, p. 1130011313, 1993.

[25] G Xie, J Luo, D Guo, and S Liu, "Nanoconfined ionic liquids under electric fields," App. Phy. Letts., vol. 96, p. 043112, 2010.

[26] H Xiao, D Guo, S Liu, G Pan, and X Lu, "Film thickness of ionic liquids under high contact pressures as a function of alkyl chain length," Trib. Letts., vol. 41, pp. 471-477, 2011.

[27] I. S. Y. Ku, J. H. Choo, T. Reddyhoff, A. S. Holmes, and H. A. Spikes, "A Novel Tribometer for the Measurement of friction in MEMS," Trib. Int., vol. 43, pp. 1087-1090, 2010.

[28] V. P. Gavrilenko, Yu. A. Novikov, A. V. Rakov, and P. A. Todua, "Measurement of Thickness of Native Silicon Dioxide with a Scanning Electron Microscope," in SPIE NanoScience Engineering, 2009, pp. 740507-740507.

[29] I. S. Y. Ku, "Lubrication of High Sliding Micromachines," Imperial College London, PhD thesis 2011.

[30] T. Reddyhoff, I. S. Y. Ku, A. S. Holmes, and H. A. Spikes, "Friction Modifier Behaviour in 
Lubricated MEMS Devices ," Trib. Lett., vol. 41, pp. 239-246, 2011.

[31] B. J. Briscoe and D. C. B. Evans., "The shear properties of langmuir-blodgett layers," Proc. Roy. Soc., Series A, Math and Phy Sci, vol. 380, no. 1779, pp. 389-407, 1982.

[32] M. He, A. S. Blum, G. Overney, and R. M. Overney, "Effect of interfacial liquid structuring on the coherence length in nanolubrication.," Phy. Rev. Lett., vol. 88, no. 15, p. 154302, 2002.

[33] S. Granick, L. Demirel, L. L. Cai, and J. Peanasky, "Soft matter in a tight spot: nanorheology of confined liquids and block copolymers," Isr. J. Chem., vol. 35, p. 3276, 1995.

[34] R. Lim and S. J. O'Shea, "Solvation forces in branched molecular liquids," Phys. Rev. Lett, vol. 88, p. 246101, 2002.

[35] M. Teodorescu, S. Balakrishnan, and H. Rahnejat, "Physics of ultra-thin surface films on molecularly smooth surfaces," Proc. IMechE, Part N: J. Nanoengineering and Nanosystems, vol. 220, pp. 7-19, 2006.

[36] J. N. Israelachvili et al., "Recent advances in the surface forces apparatus (SFA) technique," Rep. Prog. Phys., vol. 73, p. 036601, 2010.

[37] H. K. Christenson, "Interactions between hydrocarbon surfaces in a nonpolar liquid: effect of surface properties on solvation forces," J. Phys. Chem, vol. 90, pp. 4-6, 1986.

[38] J. N. Israelachvili, S. J. Kott, M. L. Gee, and T. A. Witten, "Forces between mica surfaces across hydrocarbon liquids: effects of branching and polydispersity," Macromolecules, vol. 22, p. 4247, 1989.

\section{Nomenclature}

$k_{B} \quad$ Boltzmann constant $(0.008314 \mathrm{~kJ} / \mathrm{mol} / \mathrm{K})$

$v \quad$ Sliding velocity $(\mathrm{m} / \mathrm{s})$

$v_{0} \quad$ Characteristic velocity $(\mathrm{m} / \mathrm{s})$

$E \quad$ Barrier height $(\mathrm{kJ} / \mathrm{mol})$

$P \quad$ Contact pressure $(\mathrm{Pa})$

$Q \quad$ Process activation energy $(\mathrm{kJ} / \mathrm{mol})$

$T \quad$ Contact temperature (K)

$\phi \quad$ Shear stress activation volume $\left(\mathrm{m}^{3} / \mathrm{mol}\right)$

$\tau \quad$ Shear stress $(\mathrm{Pa})$

$\tau_{0} \quad$ Pressure dependent Eyring shear stress $(\mathrm{Pa})$

$\tau_{1} \quad$ Sliding velocity dependent Eyring shear stress $(\mathrm{Pa})$

$\theta \quad$ Rate of change for friction at a given sliding velocity for constant applied load (-)

$\xi \quad$ Rate of change for friction at an applied load for constant sliding velocity (-)

$\Omega \quad$ Pressure activation volume $\left(\mathrm{m}^{3} / \mathrm{mol}\right)$ 